\title{
Quelques aspects de la pathogénie d'Entomophtbora coronata Cost. Kervork. pour la Fourmi-Manioc de la Guadeloupe : Acromyrmex octospinosus (Formicidae, Attini)
}

\author{
par A. KERMARREC et H. MAULEON \\ Station de Zoologie et de Lutte biologique, \\ Institut national de la Recherche agronomique, \\ 97170 Petit-Bourg, Guadeloupe
}

\section{Résumé.}

La recherche d'agents pathogènes de Fourmi-Manioc de Guadeloupe: Acromyrmex octospinosus Reich a permis de mettre en évidence une mycose à Entomophthora coronata Cost. Kervork. L'activité pathogène est évaluée et la présence d'une toxine démontrée. La résistance des conidies au dessèchement, aux rayons calorifiques et aux radiations stérilisantes est étudiée.

L'emploi de ce germe entomopathogène en lutte biologique est toutefois déconseillé en milieu tropical où il peut être susceptible de provoquer une mycose chez des Vertébrés.

\section{Summary.}

Some aspects of the pathogenicity of Entomophthora coronata Cost. Kervork. on the Guadeloupean parasol-ant : Acromyrmex octospinosus Reich. (Formicidae, Attini).

A general survey of pathogens of the Parasol-Ant of Guadeloupe led us to the study of the pathogenic action of Entomophtora coronata Cost. Kervork. It is shown that this fungus acts with toxic secretions. Conidia resistance to drying, heat and germicidal broadcastings are evaluated. However, the use of this entomopathogenous fungus could be dangerous in tropical areas where it is know to be a possible rhinophycomycosis agent for Vertebrates. 
La Fourmi-Manioc de la Guadeloupe : Acromyrmex octospinosus Reich (Formicidae, Attini) est élevée en nids artificiels dans lesquels on trouve parfois des individus recouverts d'un feutrage mycélien blanc et fin particulièrement abondant aux articulations et entre les tergites. Des isolats de ce champignon, qui semble être à l'origine de mortalités soudaines dans les conditions du laboratoire, ont donné lieu à des projections caractéristiques de spores en tubes inclinés. Une souche envoyée à l'Institut Pasteur de Paris fut déterminée comme étant : Entomophthora (= Conidiobolus) coronata Cost. Kervork.

L'étude de sa pathogénie pour cette fourmi a été abordée selon les étapes suivantes : mortalité lors du contact avec la culture; mortalité lors du contact avec les spores et mise en évidence d'une action par toxines. Les méthodes utilisées sont présentées pour chaque expérience.

Quelques données sur la rusticité de la souche antillaise de E. coronata ont été recherchées $=$ optimum thermique, résistance des spores au dessèchement et à l'irradiation aux ultra-violets, notamment.

\section{La mycose d'Acromyrmex octospinosus}

Les mycoses de fourmis sont citées rarement dans la littérature et il n'y a que peu de références concernant des Entomophthorales : Schaefer (1941), Rudakov (1962), Wuest et Turian (1967) et Turian et Wuest (1969). Seul Schaefer (1941) fait état de E. coronata sur une Camponote (Camponotus sp.) du Transvaal. En ce qui concerne les Attines, une infection à Beauveria fut décrite au Brésil sur un genre voisin : Atta sexdens Argolo Ferrao (1925) : in Gosswald (1938). Signalons encore que E. coronata fut trouvée par Prasertphon (1963) sur un autre insecte social : les termites.

Les cadavres porteurs de cette mycose ont l'abdomen distendu et une poudre blanchâtre déborde d'entre les segments. Selon Dieuzeide (1925) et Nirula (1957) les insectes à habitat souterrain atteints de mycose viennent généralement mourir en surface : la majorité des cadavres d'Acromyrmex octospinosus furent retrouvés à l'extérieur du nid atteint mais il faut probablement tenir compte des réactions à caractère prophylactique des ouvrières saines qui rejettent cadavres et malades à l'extérieur du nid.

Placées sur des milieux gélosés de culture de $E$. coronata les Fourmis-Manioc ont un comportement de déblaiement accru, conformément aux descriptions de Weber (1972) : découpe du substrat en cubes de 1 à 2 millimètres de côté et entassement de ceux-ci (fig. 1). La presence d'un fragment du champignon cultivé par la Fourmi stimule encore plus leur ardeur dans cette activité. Le contact avec la culture d'E. coronata s'accompagne également d'une nette augmentation des processus de toilettages mutuels.

La mortalité est estimée sur grandes ouvrières seulement, sur dix lots de dix individus par boîte de culture d'E. coronata âgée de cinq jours. Les grandes ouvrières 
furent retenues pour ces essais car leur emploi permet, d'une part, d'éviter a priori un manque d'homogénéité qui accroîtrait la variabilité biologique de la réponse et, d'autre part, les expériences d'infection sont ainsi réalisées sur la forme qui a les rapports les plus fréquents avec le milieu extérieur (éclaireuses, récolteuses, gardiennes).

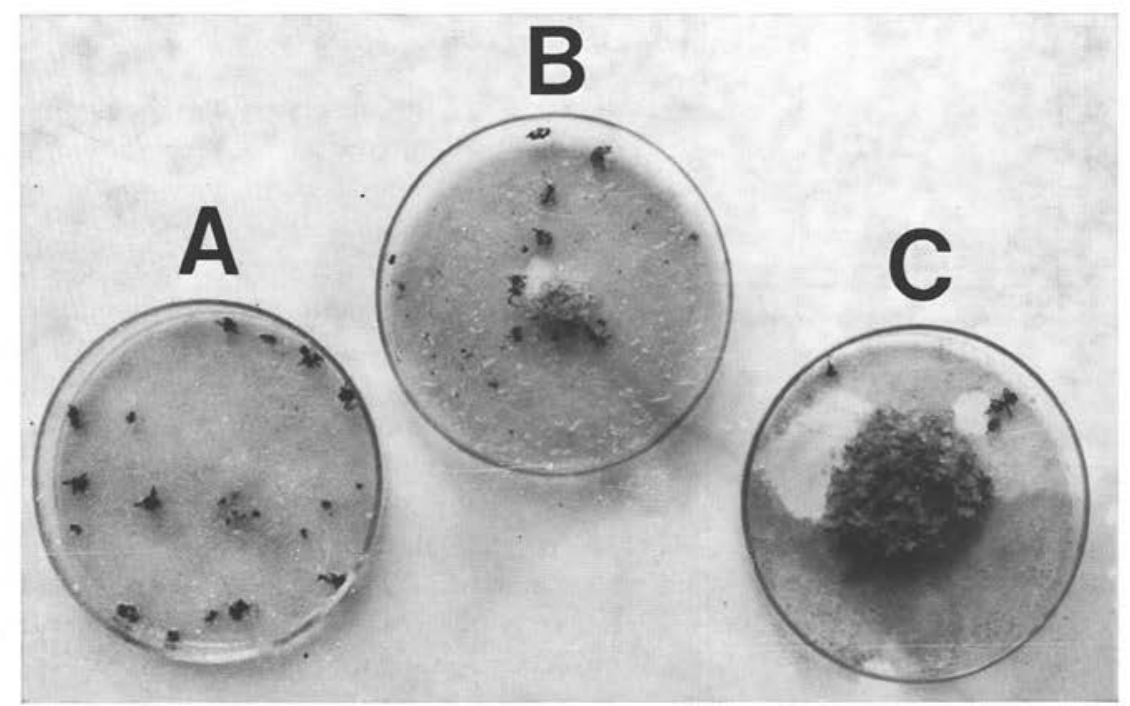

Fig. 1. - Comportement d'amassement d'A. octospinosus sur gélose contaminée par E. coronata (C) ; B : gélose contaminée par Aspergillus sp.; A : gélose témoin.

Le tableau $I$ montre la rapidité de l'action léthale de la mycose. Les fourmis placées sur la culture d'E. coronata n'ont pas survécu plus de cinq jours (TL $50 \%$ :

Tableau I. - Pourcentage de mortalité cumulée sur 100 individus en fonction de la nature du substrat sur lequel sont élevées les grandes ouvrières.

$\begin{array}{rrrr}\text { Jours } & \text { Témoin sec } & \text { Gélose avec E. coronata } & \text { Gélose s } \\ & & & \\ 0 & 0 & 0 & 4 \\ 1 & 0 & 2 & 4 \\ 2 & 3 & 20 & 22 \\ 3 & 4 & 48 & 38 \\ 4 & 6 & 100 & 42 \\ 5 & 9 & & 56 \\ 6 & 12 & & 76 \\ 7 & 16 & & 88 \\ 8 & 23 & & 98 \\ 9 & 34 & & 98 \\ 10 & 47 & & \\ 11 & 59 & & \\ 15 & 83 & & \\ 20 & 96 & & \\ 25 & 99 & & \\ 30 & 100 & & \end{array}$




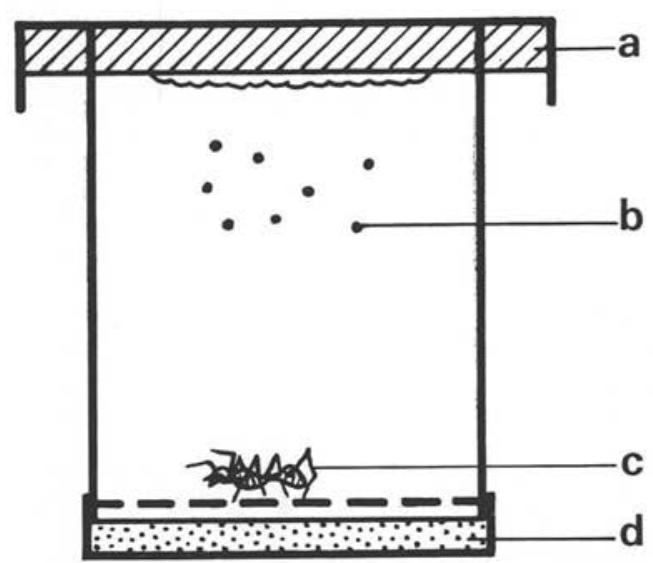

FIG. 2. - Méthode utilisée pour la mise en évidence de la pathogénie des spores: $a$ ) boîte de culture d'E. coronata; $b$ ) projection des spores; c) fourmis ; d) réservoir d'humidité.
3 jours). Les cadavres sont retirés des boîtes et placés sur un buvard humide pour vérifier le développement effectif de la mycose. Il n'y a pas de mortalité par mycose dans le témoin, mais le substrat engluant est à l'origine d'une longévité plus courte qu'en boîte à fond sec.

Le substrat utilisé pour l'expérimentation ayant des répercussions très importantes on eut recours à la technique de projection des spores (fig. 2) en milieu sec. Une boîte de culture âgée de quatre jours est retournée audessus de dix ouvrières et ceci selon cinq répétitions. La figure 3 illustre l'évolution de la mortalité induite par les conidies. Un prélèvement de spores projetées sur le fond des boîtes a mis en évidence la persistance de leur pouvoir de germination après 12 jours. La mortalité est moins rapide dans ces conditions (TL 50\%: 6 jours) que dans l'expérience précédente (TL $50 \%: 3$ jours), car les fourmis étaient alors en contact direct, dans un milieu humide, avec le mycélium, les spores et les excrétats.

D'après les expériences suivantes le mode d'action de $E$. coronata est lié à la présence de toxines excrétées :

a) Le filtrat d'une culture agitée ( 3 jours, eau $=200 \mathrm{ml}$, maltose : $10 \%$; peptone $5 \%$; pH : 8,5; $678 \mathrm{mg}$ de poids sec de $E$. coronata) est présenté en boisson à la Fourmi. La figure 4 suggère la présence d'un principe toxique per os.

b) Si l'on augmente en boîte de Pétri la dose du filtrat incorporé à l'agar des milieux il apparaît (Tableau II) que la mortalité de 100 ouvrières saines et indemnes est accélérée. Les contacts buccaux avec le milieu semblent suffire pour donner ces résultats.

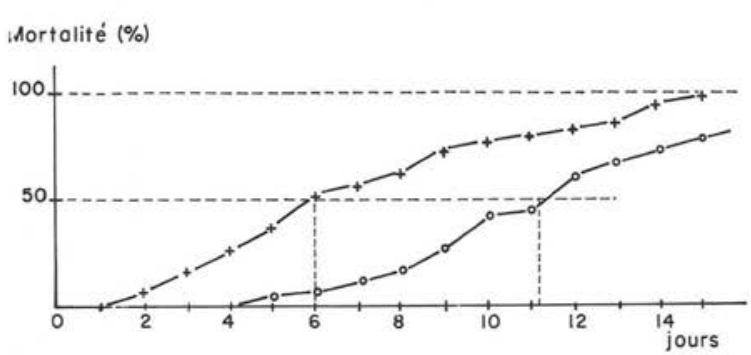

Fıg. 3. - Mortalité des ouvrières sous une pluie de spores sur substract sec et en atmosphère humide : 0 : témoins; + : traité. c) Dans ce même filtrat la Fourmi subit un bain forcé de 30 secondes. Ceci pour un lot de fourmis indemnes puis pour un lot de fourmis ayant subi, au bistouri stérilisé, une lésion atteignant l'hypoderme du thorax. D'après le Tableau III, la pénétration de la toxine dans l'hemocoele provoque une mortalité anticipée. 
TABIEAU IV. - Expansions radiales journalières moyennes de $E$. coronata sur milieu malté-peptoné à diverses températures

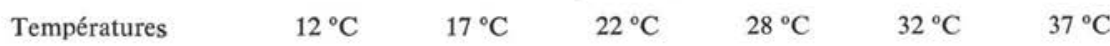

Expansion radiale $(\mathrm{mm}$ par jour) $\ldots . \ldots \ldots \ldots$ 7,5 9,0 22,5 croissances
arrêtées
18,5

La survie des conidies projetées en milieu sec a été étudiée en retournant pendant quelques heures des boîtes de culture d'E. coronata. Le couvercle, blanchi de spores est alors recouvert par un fond de boîte de Pétri sec et stérile et laissé dans l'ambiance du laboratoire : $23^{\circ} \mathrm{C}$ et $60 \%$ HR. Des prélèvements à l'aide d'un bloc d'agar de taille définie $\left(4 \mathrm{~mm}^{2}\right)$ y sont faits tous les deux jours en tamponnant (en trois endroits écartés de $120^{\circ}$ ) le couvercle afin de vérifier le maintien du pouvoir germinatif.

Au bout d'une semaine en milieu sec la conidie perd son pouvoir germinatif (Tableau V).

Tableau V. - Perte du pouvoir germinatif des conidies d'E. coronata et influence sur le retard à leur germination lors d'une exposition à $60 \% \mathrm{HR}$

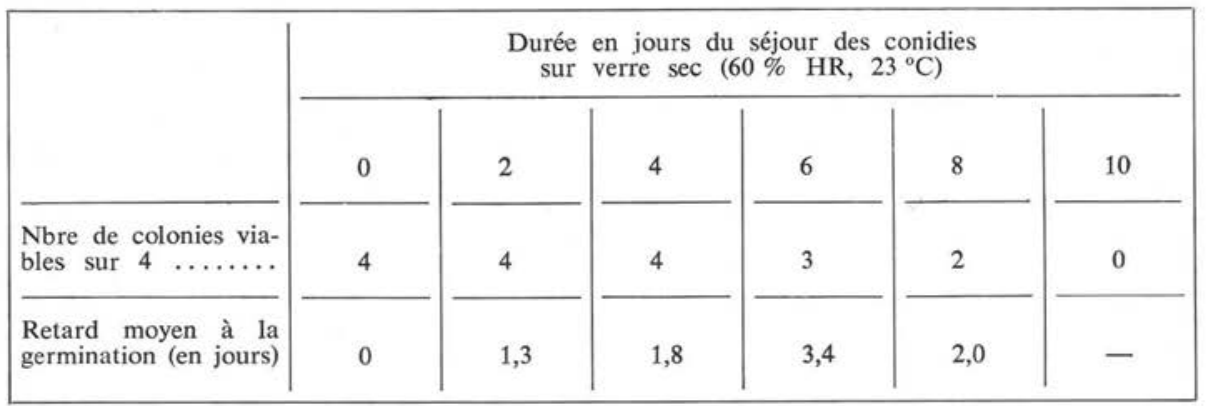

La résistance des conidies d'E. coronata aux rayons solaires stérilisants a été estimée à l'aide d'un tube à ultra-violets neuf de $40 \mathrm{~cm}$ du type \& General Electric Germicidal » émettant entre 2400 et $2600 \mathrm{~A}^{\circ}$ et placé à $50 \mathrm{~cm}$ des spores sous un réflecteur confectionné en papier aluminium ménager. Les conidies furent exposées soit sur substrat sec (verre), soit sur un milieu humide (culture). La température au niveau des conidies n'a jamais dépassé $27^{\circ} \mathrm{C}$. Les cinq prélèvements de spores à réensemencer pour vérifier leur vitalité furent effectués selon la méthode d'échantillonnage précédente et les expansions radiales moyennes des cinq cultures filles évaluées le deuxième jour de la culture (Tableau VI).

Un milieu humide protège donc mieux la spore de l'irradiation qu'un substrat sec.

La spore projetée de $E$. coronata possède une résistance d'une semaine à la dessication et une température de croissance optimale proche de la température du 
milieu subtropical antillais dont l'humidité ambiante quasi-permanente, protégerait également le germe contre les effets stérilisants d'un ensoleillement direct, occasionnel dans les sous-bois de nidification d'A. octospinosus.

TABlEAU VI. - Expansions radiales moyennes (en $\mathrm{mm}$ ) au $2^{*}$ jour suivant l'ensemencement de conidies exposées pendant des durées croissantes aux rayons stérilisants sur milieux sec et humide

\begin{tabular}{|c|c|c|c|c|c|c|}
\hline \multirow[b]{2}{*}{ Substrat } & \multicolumn{6}{|c|}{ Temps d'exposition (secondes) } \\
\hline & Témoin & 10 & 30 & 60 & 150 & 240 \\
\hline Milieu $\sec . \ldots \ldots \ldots$ & \multirow{2}{*}{17,5} & 4,0 & 3,8 & 2,5 & 1,2 & 0,0 \\
\hline Milieu humide ..... & & 13,8 & 14,0 & 11,8 & 13,0 & 9,2 \\
\hline
\end{tabular}

\section{Discussion}

En conclusion, il apparaît que $E$. coronata est un agent pathogène pour $A$. octospinosus: les spores provoquent la mort de la Fourmi et un principe toxique agit par ingestion ou par pénétration cuticulaire dans l'hémocoele selon les mêmes processus que pour d'autres insectes.

Ainsi, Yendol et al. (1968) et Prasertphon et Tanada (1969), indiquent que E. coronata et E. apiculata donnent en milieu liquide des toxines efficaces sur Galleria mellonella et Musca domestica. Jonsson (1968) précise que la quantité de toxines émises dépend de la souche et du milieu. Elles agissent après injestion ou pénétration du champignon dans l'hôte. Un travail plus récent, Yendol et Rosario (1972), compare chez Reti ulitermes flavipes (Koller) les diverses méthodes d'inoculation expérimentale qui ont été, ou pourraient être, utilisées dans l'évaluation de la virulence des Entomophthora : la méthode de la culture renversée est opposée à celle de la mise en contact direct avec la culture. Ces auteurs observent égaiement une mortalité plus rapide lors du contact direct avec le milieu de culture. L'augmentation de l'inoculum et la présence d'une mycotoxine doivent être à l'origine de cette différence.

L'intérêt théorique de cette mycose dans les conditions climatiques de la Guadeloupe, pour la lutte contre cette Fourmi est réel. Les travaux de Yendol (1968) et de Sawyer $(1929,1931)$ montraient déjà que $E$. coronata est l'espèce la plus active, et la plus tolérante aux facteurs physicochimiques du milieu, que son usage en lutte intégrée avec des insecticides est préférable à celui d'E. apiculata ou E. virulenta. Toutefois, la littérature médicale mentionne qu'en zone tropicale E. coronata peut être à l'origine de rhinophycomycoses de vertébrés. Cette phycomycose 
nasofaciale humaine, qui reste rare, fut décrite par divers auteurs (Martinson, 1963; Bras et al., 1965; Renoirte et al., 1965; Andrade et al., 1967).

Cette infection n'est cependant pas exclusive de l'homme, mais apparaît sur d'autres vertébrés : une nouvelle mycose nasale et labiale du cheval est signalée au Texas par Bridges et al. (1962), puis par Chauhan et al. (1973); la mort d'une Souris lui est imputée dans des expériences d'inhalation (Lowe et Kennel, 1972). Une issue fatale est connue à ce jour pour l'homme (Renoirte et al., 1965) et des cas humains existent en Afrique, en Inde, en Colombie, en Jamaïque, au Brésil, etc. Cette mycose tropicale frappe surtout les adultes actifs, mais n'est pas contagieuse. Une enquête menée auprès des Instituts Pasteur de la Guadeloupe et de la Guyane est restée négative quant à son incidence dans la zone française (Drouhet, comm. pers.). Le seul cas antillais connu reste donc celui de l'île Cayman au Sud de Cuba (Bras et al., 1965).

Bien que les vertébrés touchés par cette mycose présentaient probablement un terrain favorable à l'inoculum (Heimpel, 1971), il nous paraît indispensable de souligner les risques que pourraient présenter l'emploi de cette espèce d'Entomophthorale dans la lutte biologique en climat tropical.

Seuls des essais de pathogénie pour les vertébrés du type de ceux faits par Soper et Bryan (1974) avec E. thaxteriana permettraient d'apprécier la réalité du danger de la souche guadeloupéenne de $E$. coronata.

\section{REMERCIEMENTS}

Nous remercions: MM. Latgé, Remaudière, Hurpin et Ferron pour la détermination de l'agent pathogène et les conseils de rédaction du manuscrit; le $\mathrm{D}^{\mathrm{r}}$ Lefichou pour la documentation médicale; M. Messiaen pour ses conseils de mycologue et le $\mathrm{P}^{\mathrm{r}}$ Drouhet pour sa communication personnelle.

\section{Bibliographie}

Andrade (A. S.), Paula (L. A.), Sherlock (I. A.) et Cheever (A. W.), 1967. - Nasal granuloma caused by Entomophthora coronata. Am. J. Trop. Med. Hyg., 16, 31-33.

Bras (G.), Gordon (C. C.), Emmons (C. W.), Prendegast (K. M.) et Sugar (M.), 1975. - A case of Phycomycosis observed in Jamaica, infection with E. coronata. Am. J. Trop. Med. Hyg., 14, 141-145.

Bridges (C.), Romane (W.) et Emmons (C.), 1962. - Phycomycosis of horses caused by Entomophthora coronata. J. Amer. Vet. Med. Ass., 140, 673-677.

Chauhan (H. V.), Sharma (G. L.), Kabra (D. S.), Malhotra (F.C.) et Kapur (M. L.), 1973. - A fatal cutaneous granuloma due to Entomophthora coronata in a mare. Vet. Rec., 92, 425-427. 
Dieuzeide (R.), 1925. - Les champignons entomophytes du genre Beauveria (Vuillemin). Contribution à l'étude de Beauveria effusa Vuill. parasite du Doryphore. Ann. Epiphyties, 2, 185-219.

Goeswald (K.), 1938. - Über den insektentötenden Pilz Beauveria bassiana (Bals) Vuill. Bisher Bekanntes und eigene Versuch. Arb. Biol. Reich. amst., 22, 399-452.

Hall (I. N.) et Bell (J.), 1961. - Further studies on the effect of temperature on the growth of some entomophthoraceous fungi. J. Insect Pathol., 3, 289-296.

Heimpel (A. M.), 1971. - Safety of insect pathogens for man and vertebrates. In: Microbial control of Insects and Mites. Burges et Hussey, Ed., Academic Press, Publ., London and New York.

Jonsson (A. G.), 1968. - Protease production by species of Entomophthora. Appl. Microbial., 16, 450-457.

Lowe (R. E.) et KenNel (E. W.), 1972. - Pathogenicity of the fungus Entomophthora coronata in Culex pipiens quinquefasciatus and Aedes taeniorhynchus Mosq. News, 32, 614-620.

Martinson (F.), 1963. - Rhynophycomycosis. J. Laryngol. Otol., 77, 691-705.

NirULA (K. K.), 1957. - Observations on the green muscardine fungus in populations of Oryctes rhinoceros L. J. Econ. Entomol., 50, 767-770.

Prasertphon (C.), 1963. - Pathogenicity of different strains of Entomophthora coronata Cost. Kervork. for larvae of the greater wax moth Galleria mellonella L. J. Invertebrate Pathol., 5, 174-181.

Prasertphon (C.) et TANada (Y.), 1969. - Mycotoxine of Entomophthoraceous fungi. Hilgardia, $39,581-600$.

Renoirte (R.), Vandepille (J.), Gatti (P.) et Werth (R.), 1965. - Phycomycose nasofaciale (Rhinophycomycose) due à Entomophthora coronata. Bull. Soc. Pathol. Exotique, 58, 847-862.

RudaKov (O. L.), 1962. - Mikoz murav'ev (Predvant scobshchenie). Sb. entomol. rabot. akd. nauk. Kierghizsk. SSR. Lierghizsk, otb. Vses. Entomol. obshch., I, 128130.

SAWYer Jr. (W. H.), 1929. - Observations on some entomogenous members of the Fntomophthoracea in artificial culture. Amer. J. Bot., 16, 87-121.

SAWYER Jr. (W. H.), 1931. - Studies on the morphology and development of an insectdestroying fungus, Entomophtora sphaerosperma. Mycologia, 23, 411-432.

SCHAEFFer (E. E.), 1941. - A fungus of the family Entomophthoraceae found on sugar ants (Camponotus sp.). Bothalia, 4, 237-249.

Soper (R. S.) et Bryan (T. A.), 1974. - Mammalian safety of the Aphid attacking fungus Entomophthora nr. thaxteriana. Environ. Entomol., 3, 346-347.

TURIAN (G.) et Wuest (J.), 1969. - Mycoses à Entomophthoracées frappant des populations de fourmis et de drosophiles. Bull. Soc. Entomol., Suisse, 42, 197-201.

Weber (N. A.), 1972. - The fungus culturing behaviour of ants. American Zoologist, 12, 577-587. 
Wuest (J.) et Turian (G.), 1967. - Une épizootie à Tarichium (Entomophthoraceae) sur la larve de Ocnogyna parasita Hbn. (Lépid. Arcctiidae). Mitt. Schwezi. ent. Gesell., 40, 96-99.

YENDOL (W. G.), 1968. - Factors affecting germination of Entomophthora conidia. J. Invertebrate pathol., 10, 116-121.

Yendol (W. G.), Miller (E. M.) et Behnke (C. N.), 1968. - Toxic substances from entomophthoraceous fungi. J. Invertebrate Pathol., 10, 313-319.

Yendol (W. G.) et Rosario (S. B.), 1972. - Laboratory evaluation of methods for inoculating termites with entomophthoraceous fungi. J. Econ. Entomol., 65, 1027-1029. 